\title{
Cem anos de buracos negros: o centenário da solução de Schwarzschild
}

\section{One hundred years of black holes: the centenary of the Schwarschild solution}

\author{
Alberto Saa*1 \\ ${ }^{1}$ Departamento de Matemática Aplicada, Universidade Estadual de Campinas, 13083-859, Campinas, SP, Brasil
}

Recebido em 27 de Agosto, 2016. Aceito em 28 de Agosto, 2016.

\begin{abstract}
No início deste ano de 2016, o consórcio formado pelas colaborações LIGO e Virgo anunciou a primeira detecção direta de ondas gravitacionais. Exatamente 100 anos antes, Karl Schwarzschild, um físico e astrônomo alemão de refinada formação matemática, surpreendia Einstein e toda a comunidade científica com uma solução exata das complicadas equações da Teoria da Relatividade Geral. As condições nas quais desenvolveu este seu trabalho foram dramáticas e ficaram famosas: lutando com o exército alemão na frente russa da Primeira Guerra Mundial. É notável também que seu trabalho tenha sido praticamente simultâneo aos de Einstein que levaram à consolidação da Relatividade Geral, a tempo ainda de ter sua solução reconhecida e citada na formulação definitiva da Teoria, publicada por Einstein no mesmo ano de 1916. A desde então chamada solução de Schwarzschild foi o ponto de partida para uma das mais interessantes sagas da Física do Século XX: a descoberta dos buracos negros e suas fascinantes propriedades físicas, que estão por trás, também, dos fenômenos de ondas gravitacionais detectados diretamente este ano. Apresentaremos aqui um pouco dos contextos histórico e epistemológico destas notáveis descobertas e alguns de seus principais protagonistas.
\end{abstract}

Palavras-chave: Relatividade Geral, Buracos Negros, Ondas Gravitacionais, Karl Schwarzschild

Early this year, 2016, the consortium formed by LIGO and Virgo collaborations announced the first direct detection of gravitational waves. Exactly 100 years earlier, Karl Schwarzschild, a German physicist and astronomer with a refined mathematical background, surprised Einstein and the entire scientific community with an exact solution for the complicated equations of General Relativity. The conditions under which he worked on the problem were quite dramatic and became famous: fighting with the German army on the Russian front of the First World War. It is remarkable also that his work was almost simultaneous to Einstein's that led to the consolidation of General Relativity, just in time to have his solution recognized and cited in the final formulation of the Theory, published by Einstein in the same year of 1916. Since then called the Schwarzschild solution, it was the starting point for one of the most interesting sagas of the twentieth century Physics: the discovery of black holes and their fascinating physical properties, which are also behind the gravitational waves phenomena directly detected this year. We present here a bit of the historical and epistemological contexts of these remarkable discoveries and some of their main protagonists.

Keywords: General Relativity, Black Holes, Gravitational Waves, Karl Schwarzschild

\section{Introdução}

Em fevereiro de 2016, o consórcio formado pelas colaborações LIGO [1] e Virgo [2] anunciou, com grande interesse e cobertura dos principais meios de comunicação, a primeira detecção direta de ondas

*Endereço de correspondência: asaa@ime.unicamp.br gravitacionais [3 6], culminando assim de maneira esplêndida mais de meio século de esforços teóricos e tecnológicos fundamentais para esta façanha observacional, considerada por muitos o embrião de uma nova e fascinante era na observação astronômica [4]. Detecções mais frequentes são esperadas no futuro (de fato, um outro evento semelhante ao primeiro já 
foi detectado 7$]^{1}$ e, com isso, dados observacionais fundamentais para o entendimento dos fenômenos físicos envolvidos passarão a ser acumulados em bom ritmo, permitindo desta maneira um cuidadoso escrutínio das teorias físicas pertinentes, levando ao eventual descarte de algumas hipóteses e o aprimoramento de outras, como de fato se espera da dinâmica do Método Científico nas Ciências Experimentais. Há grande expectativa na comunidade científica sobre estas novas massas de dados e a natureza dos eventos e fenômenos físicos que poderão ajudar a elucidar.

Os fenômenos físicos por trás das emissões de ondas gravitacionais de detecção mais provável como, em particular, é justamente o caso dos dois eventos já observados, envolvem buracos negros, mais especificamente situações de fusão entre dois buracos negros. São fenômenos tipicamente de energias gigantescas, são de fato os mais energéticos, depois do Big Bang, conhecidos na Física, nos quais massas de mesma ordem de magnitude que a do sol são convertidas e irradiadas como ondas, com predominância das gravitacionais. Tanto os buracos negros quanto as ondas gravitacionais são previsões originais da Teoria da Relatividade Geral, construída também há um século por Albert Einstein $2^{2}$ para a descrição dos fenômenos gravitacionais, substituindo, até agora com absoluto sucesso, o paradigma da Gravitação Universal de Newton. Não havia, até este ano, nenhuma observação direta de ondas gravitacionais, mas também é certo que já havia sido acumulado um considerável conjunto de evidências convincentes a favor de sua existência. Por exemplo, o prêmio Nobel de 1993 [10 foi concedido a R. Hulse e J. Taylor precisamente pelas observações realizadas nos anos 70 sobre um sistema binário formados por um pulsar e uma estrela de nêutrons [11]. Das ondas de rádio emitidas por este sistema binário, é evidente que ele vem perdendo energia, com uma taxa de decréscimo perfeitamente compatível com as previsões da Relatividade Geral sobre a emissão

\footnotetext{
${ }^{1}$ Os dois eventos detectados foram denominados GW150914 e GW151226. GW denota o tipo do evento (gravitational wave), e o número seguinte a data da detecção: ano, mês e dia. O anúncio da detecção é sempre posterior, alguns meses são necessários para a completa análise do sinal capturado. Os dados dos sinais são públicos. Os do primeiro evento, por exemplo, podem ser obtidos no endereço https://losc.ligo.org/events/GW150914/

${ }_{2}$ Para uma biografia de Einstein, recomenda-se 8. Como texto de divulgação sobre a Relatividade Geral e sua rica história, recomenda-se 9 .
}

de ondas gravitacionais por sistemas dessa natureza. Sobre os buracos negros especificamente, ainda não há observação direta, mas a detecção das ondas gravitacionais possivelmente oriundas da fusão de buracos negros é, incontestavelmente, mais uma forte evidência indireta de sua existência na Natureza. Retornaremos a este ponto mais adiante.

Uma efeméride notável nos remete precisamente à origem dos buracos negros, objetos astrofísicos de propriedades surpreendentes e história rica em controvérsias, polêmicas e curiosidades. Exatos 100 anos antes do anúncio da primeira detecção direta de ondas gravitacionais, em fevereiro de 1916, era publicado nos Anais da Academia Real de Ciências da Prússia um artigo, correspondente a uma apresentação oral feita por Einstein em 13 de janeiro desse mesmo ano, contendo um resultado surpreendente obtido por um físico e astrônomo alemão de refinada formação matemática: Karl Schwarzschild. Neste trabalho 12$]^{3}$ intitulado "Sobre o campo gravitacional de uma massa puntual de acordo com a teoria de Einstein", Schwarzschild apresenta a solução que viria a ser imortalizada com o seu nome para as equações da Relatividade Geral de Einstein, e que levará não só à descoberta dos buracos negros, mas como também a boa parte da Física que hoje está sendo desvendada com as observações das ondas gravitacionais. Nos seus 100 anos de história, a solução de Schwarzschild foi intensamente debatida física e matematicamente, caiu no esquecimento, renasceu, foi completamente dissecada e generalizada, até chegarmos no entendimento atual de que ela descreve o tipo mais simples de uma nova classe de objetos da Natureza: os buracos negros. Serão apresentados a seguir um pouco dos contextos histórico e epistemológico nos quais se desenvolveu esta que certamente é uma das grandes sagas da Física do século XX: a descoberta dos buracos negros e suas fascinantes propriedades físicas. Iniciaremos com uma breve biografia científica do principal personagem da efeméride.

\section{Karl Schwarzschild}

Karl Schwarzschild nasceu em uma família judaica de alto estrato sócio-cultural da cidade de Frankfurt, Alemanha, em 9 de outubro de 1873, sendo

\footnotetext{
${ }^{3}$ Vários dos trabalhos já clássicos em língua alemã citados aqui podem ser encontrados em versão original no projeto Wikisource http://de.wikisource.org/
} 
o primogênito de seis irmãos [13,14]. Foi um estudante brilhante e demonstrou precocemente seu talento para a Matemática e as Ciências Naturais. Seus dois primeiros trabalhos científicos 15, 16, sobre mecânica celeste, foram publicados antes de seu décimo sétimo aniversário, enquanto ainda era um estudante pré-universitário em sua cidade natal. Nessa época, aventurava-se como auto-didata junto a um amigo dois anos mais velho, Paul Epstein, que viria posteriormente a se consagrar como um grande matemático, em diversos tópicos de Matemática superior e astronomia, incluindo a observação do céu com telescópios.

Schwarzschild frequentou entre 1891 e 1893 a Universidade de Estrasburgo, então uma Universidade alemã geográfica e culturalmente, onde teve uma formação sólida em astronomia, incluindo técnicas observacionais modernas e pioneiras na época. Em seguida, muda-se a Munique, onde realiza um ano de serviço militar em um corpo de artilharia do exército alemão. Sob orientação do eminente astrônomo Hugo von Seeliger, obtém em 1896, na Universidade de Munique, seu doutorado [17], explorando alguns dos trabalhos matemáticos de Poincaré e suas possíveis aplicações à dinâmica e origem do sistema solar. Sua refinada formação matemática é evidente em seus trabalhos dessa época. Seu doutorado, por exemplo, envolve ideias matemáticas introduzidas e desenvolvidas por H. Poincaré poucos anos antes, acerca de estabilidade de fluidos auto-gravitantes em rotação. Logo após obter seu doutorado, assumiu uma posição de astrônomo no Observatório Kuffner, de Viena, onde ficou até 1899 , ano em que regressa a Munique com uma tradicional posição acadêmica da época na Alemanha: a de Privatdozent. Seu exame de Habilitação, necessário pelas regras das universidades alemãs para se ter acesso a essa posição, será importante, como veremos, para entendermos seu grau de familiaridade, anos depois, com a Relatividade Geral. De seu período em Viena, destacam-se seus trabalhos teóricos sobre o Problema de Três Corpos e, principalmente, seus trabalhos sobre fotometria, técnica pioneira na época e que provou ser fundamental para o desenvolvimento da astronomia moderna.

Schwarzschild deixa Munique em 1901, ano em que assume uma posição de Professor na universidade que provavelmente era a de maior prestígio em Física e Matemática da Alemanha na época: Göttingen, onde fica até 1909, sendo o responsável pelo

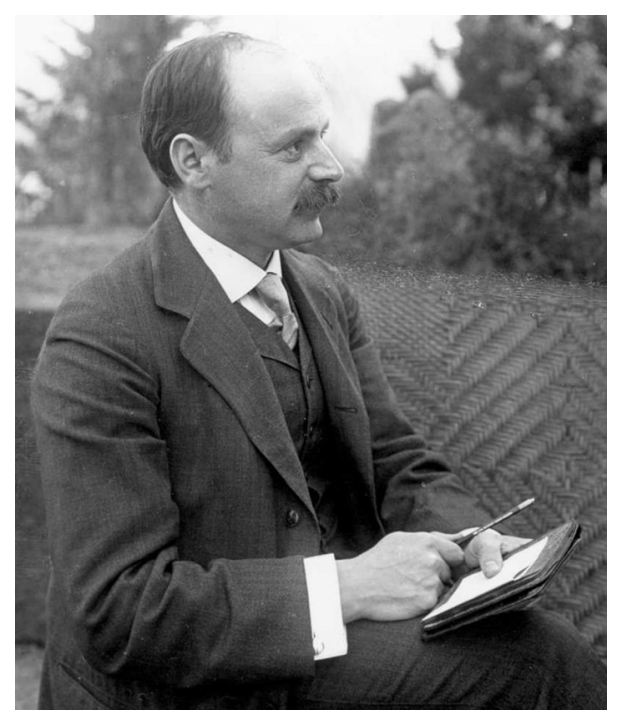

Figura 1: Karl Schwarzschild, 9 de Outubro de 1873 (Frankfurt) - 11 de Maio de 1916 (Potsdam), em seus anos na Universidade de Göttingen $(1901$ - 1909). Imagem de domínio público, https://commons.wikimedia.org/

Observatório Astronômico da Universidade. Teve como colegas e colaboradores nesse período a Felix Klein, David Hilbert e Hermann Minkowski, o que certamente foi importante para mantê-lo atualizado constantemente sobre os avanços na Matemática e o desenvolvimento da Teoria da Relatividade Geral de Einstein. Em Göttingen, continua trabalhando em Física Teórica e também realiza diversas observações astronômicas, produzindo um extenso catálogo de estrelas: o Göttinger Aktinometrie. Em 1909, mudase para Potsdam, onde assume a posição de maior destaque na Alemanha da época para um astrônomo: a de Diretor do Observatório Astrofísico da cidade. É durante esse período que forma sua família. Casase com Else Posenbach em seus últimos meses em Göttingen, vindo a ter três filhos, já em Potsdam: Agathe, Martin e Alfred. Seu filho Martin Schwarzchild deixará a Alemanha durante a perseguição nazista, e desenvolverá sua carreira de grande sucesso como astrônomo na Universidade de Princeton, nos Estados Unidos.

Em Potsdam, Schwarzchild prossegue com seus trabalhos astronômicos de primeira classe. Continuou a contribuir para o grande catálogo de estrelas iniciado em Göttingen. Visitou os Estados Unidos em 1910, participando no (décimo primeiro) Congresso da AAS (American Astronomical Society), em Cambridge, Massachusetts, e na (quarta) Conferência da International Union for Solar Research, em Mount Wilson, Califórnia. Ainda em 1910, 
participa da expedição alemã a Tenerife, nas Ilhas Canárias, para a observação da passagem do cometa Halley, registrando o evento com suas técnicas fotográficas pioneiras na época. Contudo, sua carreira brilhante na astronomia foi bruscamente interrompida em 1914, com a eclosão da Primeira Guerra Mundial. Schwarzchild, que estaria dispensado de se unir às filas militares por sua idade, se apresenta como voluntário, trabalhando ativamente no esforço de guerra alemão, primeiro como oficial de meteorologia e, posteriormente, de artilharia. Chega, inclusive, a produzir trabalhos científicos de caráter eminentemente bélico, como, por exemplo, um estudo sobre a influência da resistência do ar na trajetória de projéteis balísticos [18, o qual devido à guerra só veio a ser publicado em 1920. Há uma vasta e rica literatura 4 sobre essa conturbada e extremamente trágica época da sociedade alemã, na qual inúmeros cientistas de primeira magnitude eram verdadeiros entusiastas da guerra 20]. Muitos viriam a pagar o mais alto preço por essas escolhas. Há de se destacar, sempre, que um dos raros cientistas alemães que se manifestaram enfaticamente contra a guerra foi Albert Einstein [8]. Schwarzschild serviu inicialmente em Namur, Bélgica, como oficial de meteorologia, a partir de setembro de 1914 (a guerra se iniciara em 28 de julho). Depois, passou rapidamente pela região de Argonne, França, e finalmente, já como oficial de artilharia, foi para a temida e perigosa frente russa. O grande físico indiano Subrahmanyan Chandrasekhar, do qual em breve falaremos, ganhador do Prêmio Nobel de 1983 por seus estudos fundamentais sobre estrutura e evolução estelar, relata uma conversa nos anos 30 com o matemático alemão Richard Courant, que viria a se estabelecer nos Estados Unidos após a perseguição nazista, na qual ele dizia ter, com muita surpresa, encontrado Schwarzschild a caminho da frente russa, enquanto ele próprio a abandonava. Nas próprias palavras de Chandrasekhar: 14

Richard Courant told me in the late thirties that he had met Karl Schwarzschild proceeding to the Eastern front while he, as a member of the general staff, was with a party retreating from the same front; and Courant said that he was surprised that someone as distinguished as Karl Schwarzschild would be proceeding

\footnotetext{
$\overline{{ }^{4} \text { Ver, por exemplo, } \sqrt{19}}$ e referências lá contidas.
}

\section{towards a front that was considered too dangerous for the general staff!}

Durante a campanha russa, Schwarzschild contraiu pênfigo, uma debilitante doença auto-imune que causa bolhas e erupções extremamente dolorosas na pele, completamente incurável na época. Foi desmobilizado em março de 1916, vindo a morrer dois meses depois. Não chegou a completar 43 anos de idade.

\subsection{A solução e seu contexto}

Schwarzschild também se dedicou à Ciência Básica enquanto esteve mobilizado no exército alemão. Foi provavelmente no verão de 1915, já na frente russa, quando desenvolveu os trabalhos que deram origem a seus dois artigos sobre relatividade. Além do primeiro já comentado anteriormente [12], houve um segundo [21] sobre o campo gravitacional de uma distribuição esférica e homogênea de matéria. É neste segundo artigo que aparece, pela primeira vez, a quantidade $r_{S}$, que passaria a ser chamada "raio de Schwarzschild". O ano de 1915 foi também particularmente intenso para Einstein. Publicou quatro artigos em novembro [22 25], os quais viriam a ser a base da sua Teoria da Relatividade Geral. Curiosamente, o artigo final, consolidando a teoria, só foi publicado em março de 1916 [26], após o primeiro trabalho de Schwarzschild, o qual é de fato citado, e portanto inequivocamente reconhecido como válido e pertinente, por Einstein. Em um de seus quatro trabalhos de Novembro [24], Einstein propõe, como se infere literalmente do título, uma "explicação para o movimento do periélio de Mercúrio a partir da Teoria da Relatividade Geral". Trata-se de um dos testes clássicos da Relatividade Geral. Sabe-se, das Leis de Kepler, que a órbita dos planetas é uma elipse com o Sol situado em um de seus focos. Os nomes de periélio e afélio são dados, respectivamente, aos pontos da órbita de menor e maior distância ao sol. São, obviamente, os pontos extremos do eixo maior da elipse correspondente à órbita em questão. A Teoria da Relatividade Geral prevê um pequeno movimento, um avanço, do periélio de Mércurio, que provou ser perfeitamente compatível com as observações $5^{5}$ A órbita de Mercúrio é, portanto, uma elipse que precessa em torno do sol, e

\footnotetext{
${ }^{5}$ Rigorosamente, a previsão da Relatividade Geral que foi confirmada nas observações foi de um excesso de precessão, já que o a precessão em si também é prevista no contexto newtoniano, a partir da perturbação da órbita de Mercúrio
} 
este movimento pode ser detectado registrando-se a posição do periélio a cada volta completa (ano) do planeta em torno do sol. O planeta Mercúrio é particularmente útil para este estudo por dois principais motivos [27]: é o planeta com maior velocidade de translação em torno do Sol, com um ano que corresponde aproximadamente a 88 dias do planeta Terra, sendo possível portanto registrar a posição de seu periélio a cada três meses terrestres; e também por ser o planeta com a órbita de maior excentricidade (0.206) do sistema solar, depois de Plutão (0.248), que de fato não é mais considerado um planeta. A excentricidade da órbita da Terra, por exemplo, não chega a um décimo da de Mercúrio.

Para a derivação de sua previsão para o avanço do periélio de Mercúrio, Einstein deveria descrever o equivalente em sua Teoria da Relatividade Geral do campo gravitacional do Sol. Suas equações, porém, se mostravam muito mais complicadas que as da Gravitação Universal de Newton, e Einstein então optou por uma abordagem perturbativa. Não obteve a solução exata que corresponderia na Relatividade Geral ao campo gravitacional de um corpo esférico, mas descreveu as correções oriundas da Relatividade Geral ao potencial Newtoniano, o qual, para o caso de um corpo esférico e homogêneo de massa $M$ situado na origem das coordenadas cartesianas $(x, y, z)$ é dado pela simples expressão

$$
\Phi(x, y, z)=-\frac{G M}{\sqrt{x^{2}+y^{2}+z^{2}}},
$$

sendo $G$ a constante universal de Newton. O potencial newtoniano (1) é válido para a região externa ao corpo. No entanto, essa expressão não envolve o tamanho do corpo. Em outras palavras, é válida para corpos esféricos e homogêneos de qualquer raio, inclusive os arbitrariamente pequenos, i.e., corpos puntuais ${ }^{6}$

Em seu primeiro artigo de 1916 [12, Schwarzschild encontra a solução exata das equações de Einstein

devido à presença de outros planetas, notadamente Júpiter. Para se ter uma ideia, as previsões Newtonianas são da ordem de 530 segundos de arco por século, enquanto as da Teoria da Relatividade Geral não atingem 1/10 disso (43"/século). ${ }^{6}$ De fato, este é o conteúdo da $71^{\text {a }}$ Proposição da Seção 12 do Principia de Newton 28], provado como o Teorema 31. Nas palavras de Newton (na versão inglesa):

... a corpuscle placed with out the sphaerical superficies is attracted towards the centre of the sphere with a force reciprocally proportional to the square of its distance from that centre. que corresponderia ao campo gravitacional de uma massa puntual. Eu outras palavras, o equivalente na Relatividade Geral ao potencial (1) na Gravitação Universal de Newton. Sua solução confirmou que as aproximações feitas por Einstein em seu trabalho sobre o periélio de Mercúrio estavam de fato corretas. Schwarzschild enviou o resultado a Einstein em uma famosa carta, em dezembro de 20157 A reação de Einstein foi de entusiasmo. Em sua resposta, escrita em um cartão postal em 29 de Dezembro, escreveu

\section{Ich hätte nicht gedacht, dass die strenge} Behandlung des Punktproblems so einfach wäre 8

Einstein também prometeu uma publicação rápida do resultado. Em janeiro de 1916, faz a comunicação no encontro da Academia Real de Ciências da Prússia, e o artigo é de fato publicado nos Anais de fevereiro.

A Relatividade Geral é uma teoria radicalmente diferente da Gravitação Universal de Newton. Em particular, a interação gravitacional não é mediada por um potencial como (1), mas pelas propriedades geométricas do Espaço-Tempo, conceito fundamental que havia sido introduzido em 1908 pelo colega de Schwarzschild em Göttingen H. Minkowski 30. As propriedades geométricas em questão são as descritas pelo chamado tensor métrico, com o qual pode-se escrever o equivalente de uma noção infinitesimal de comprimento para o Espaço-Tempo, o chamado elemento de linha. Curiosamente, Schwarzschild escreve sua solução já na forma "moderna" em coordenadas espaço-temporais esféricas $(t, r, \theta, \phi)$, nas quais o elemento de linha da solução é dado por

$$
d s^{2}=\left(1-\frac{r_{S}}{r}\right) d t^{2}-\frac{d r}{1-\frac{r_{S}}{r}}-r^{2}\left(d \theta^{2}+\operatorname{sen}^{2} \theta d \phi^{2}\right),
$$

mas sua interpretação correta ainda tardaria. Em seu segundo trabalho [21, publicado em abril de 1916, no qual Schwarzschild considera não o caso de um massa puntual, mas uma distribuição esférica e homogênea de matéria, sua constante $r_{S}$ é finalmente identificada como

$$
r_{S}=\frac{2 G M}{c^{2}},
$$

sendo $c$ a velocidade da luz no vácuo. Desse trabalho, pode-se já inferir que não será possível um

\footnotetext{
${ }^{7}$ A carta original e sua resposta estão reproduzidas em 14 . Ver também: 29

${ }^{8}$ Não imaginei que a solução exata para o problema puntual fosse tão simples - tradução livre do autor.
} 
equilíbrio hidrostático se a distribuição de matéria tiver um raio inferior a $\frac{9}{8} r_{S}$. Este ponto, que de fato é o embrião da ideia dos buracos negros, não foi completamente percebido e portanto explorado por Schwarzschild. Esta questão será melhor exposta a seguir.

É bastante surpreendente que Schwarzschild tenha enviado a Einstein sua solução em 22 de dezembro de 1915, pouco mais de um mês após a publicação dos quatro artigos preliminares de Einstein sobre a Relatividade Geral 22 25], principalmente levando-se em conta que Schwarzschild estava em pleno campo de batalha na Rússia. Seria uma façanha virtualmente impossível para alguém sem uma notável familiaridade com a Matemática de ponta na época e com as ideias da Relatividade Geral. Ocorre que Schwarzschild tinha a formação necessária para contribuir em pé de igualdade com Einstein para a Relatividade Geral. Não se trata de um exagero retórico. Em seus anos de Göttingen, teve como colegas e colaborou com Klein, Hilbert e Minkowski, três matemáticos de fundamental importância para o desenvolvimento da Física e da Matemática dos fenômenos gravitacionais. Minkowski, em particular, fora o responsável anos antes pela introdução da ideia de Espaço-Tempo [30. Hilbert trabalhou ativamente nos fundamentos matemáticos que possibilitaram a derivação das equações da Relatividade Geral. É importante destacar que Einstein, muito famoso pela economia em suas citações científicas, inclui apenas duas referências em seu trabalho definitivo sobre a Relatividade Geral [26]: o primeiro artigo de Schwarzschild e um de Hilbert. Felix Klein foi fundamental no desenvolvimento das chamadas geometrias não-euclidianas, conceitos fundamentais no desenvolvimento da Física e da Matemática do Século XX, sendo, em particular, um dos pilares da Relatividade Geral.

A ideia de que outras geometrias além da milenar euclidiana poderiam fazer sentido foi elaborada em sua forma moderna por Bernhard Riemann, que este ano tem o sesquicentenário de sua morte relembrado [31]. Assim como Schwarzschild (e Minkowski), teve uma vida breve, menos de 40 anos, mas fundamental para o desenvolvimento da Matemática e da Física. Schwarzschild tinha intimidade com a obra de Riemann antes mesmo de seus dias de Professor em Göttingen, antes de ser colega de Klein, Hilbert e Minkowski. Em seu exame de Habilitação, no ano de 1899, em Munique, Schwarzschild apresenta uma monografia sobre suas contribuições à fotometria e suas aplicações à astronomia, a qual é aprovada. Porém, dentre os temas que haviam sido considerados para sua dissertação, estava uma proposta de verificação, a partir de observações astronômicas, da natureza geométrica do espaço [14]. Trata-se de uma proposta de um arrojo intelectual incrível: a de que as geometrias não-euclidianas que faziam sentido matematicamente poderiam existir de fato na Natureza, e Schwarzschild apresenta uma proposta científica para verificar esta possibilidade! Incentivado por Seeliger, apresenta este trabalho 32 no encontro anual da Sociedade Astronômica Alemã em Heidelberg, em 1900, cinco anos antes do "ano miraculoso" em que Einstein apresenta a teoria da Relatividade Especial, e 15 anos antes da Teoria da Relatividade Geral! Nesse fantástico trabalho, Schwarzschild mostra que os dados astronômicos disponíveis na época limitariam os possíveis raios de curvatura para o espaço tri-dimensional. Seriam, no mínimo, o equivalente a 4 milhões de unidades astronômicas 9 se o espaço fosse do tipo hiperbólico, ou 100 milhões se o espaço fosse esférico. Muito mais importante que esses números, que não são de todo precisos, era a ideia de testar experimentalmente a geometria realizada na Natureza. Há uma ampla literatura, na fronteira com a Filosofia, sobre este assunto, cobrindo grande parte da tradição acadêmica alemã, notadamente os trabalhos de Gauss [33] e as referências aos ideais kantianos 34]. Não há dúvidas que Schwarzschild esteve na fronteira do conhecimento humano em seus dias.

\subsection{Johannes Droste}

Antes de passarmos a uma discussão sobre o conteúdo físico da solução de Schwarzschild (2), é interessante comentar um caso emblemático sobre o surgimento de novas ideias na Física e na Matemática, e o mecanismo de atribuição dos devidos créditos. Trata-se do caso do físico e matemático holandês Johannes Droste [35]. O notável neste caso é que Droste obtém a mesma solução de Schwarzschild, quase que simultaneamente! Seu artigo [36], intitulado "O campo de um centro único na Teoria de Einstein da Gravitação, e o movimento de uma partícula nesse campo" apresenta exatamente a solução de Schwarzschild (2). Este trabalho foi apresentado pelo então orientador

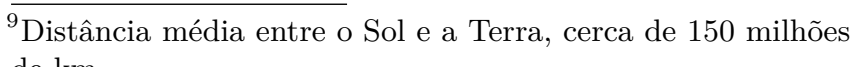
de $\mathrm{km}$. 
de Droste, Hendrik Lorentz, no encontro da Academia Holandesa de Ciências em 27 de maio de 1916. Neste trabalho, publicado paralelamente ao original holandês também em língua inglesa, lê-se a seguinte nota de rodapé:

After the communication to the Academy of my calculations, I discovered that also K. Schwarzschild has calculated the field. Vid: Sitzungsberichte der Kon. Preuss. Akad. der Wiss. 1916, page 189, Equation (7) agrees with (14) there, if $R$ is read instead of $r$.

Pouco se sabe sobre Johannes Droste [37. Nasceu em Grave, Holanda, em 28 de maio de 1986 e faleceu em Leiden, em 16 de setembro de 1963. Obteve seu doutorado em Leiden em 1916, com uma tese exatamente sobre campos gravitacionais na Teoria da Relatividade Geral [38]. Aparentemente, foi a única tese em Relatividade Geral orientada por Lorentz. De seu trabalho [36], infere-se que vinha trabalhando com Relatividade Geral desde 1913, ano em que Einstein publica alguns artigos bastante preliminares sobre a teoria. Assim como no caso de Schwarzschild, é evidente que Droste tinha um bom domínio dos aspectos matemáticos e físicos da Relatividade Geral para obter esses resultados originais. Muito provavelmente, tinha também condições de contribuir em pé de igualdade com os outros pioneiros da Relatividade Geral. Sua derivação da solução em [36 é mais clara que a de Schwarzschild. Sua análise do movimento de partículas de teste nesse campo gravitacional, além de ser mais completa que a de Schwarzschild, é de grande originalidade, chegando a adiantar em quase 50 anos as chamadas "coordenadas tartarugas", essenciais para o entendimento da Física dos buracos negros. Chega também a identificar corretamente algumas das propriedades das órbitas circulares com raio $r=3 r_{S}$, chamadas atualmente de Innermost stable circular orbi110. Em vários aspectos, o trabalho de Droste é mais profundo que o de Schwarzschild. Apesar disso, seu trabalho caiu no completo esquecimento, mesmo com os esforços dos que advogam [35, 37] a favor da utilização da expressão Schwarzschild-Droste para denotar a solução (2). Os motivos pelos quais Droste não teve o merecido reconhecimento são, infelizmente, mais comuns do

${ }^{10}$ ISCO, normalmente traduzida em português como "última orbita circular estável". que gostaríamos no mundo científico. O resultado de Schwarzschild, então diretor do mais importante observatório alemão, ainda que afastado pela guerra, fora comunicado por ninguém menos que Albert Einstein, num encontro da mais prestigiosa academia da época. Droste era então um estudante de doutorado pouco conhecido. Ainda que seu orientador era um renomado físico e que a academia holandesa de ciências gozava de grande prestígio, não eram páreo para Einstein e para a Academia Prussiana. O trabalho de Schwarzschild é imediatamente reconhecido e passa a ser citado, sendo a sua primeira citação nada mais nada menos do que uma das duas únicas referências no trabalho definitivo de Einstein sobre a Relatividade Geral [26], enquanto o trabalho holandês cai no ostracismo. A partir de 1916, Droste se dedicou mais à Matemática, tendo ocupado uma posição de Professor na Universidade de Leiden por muitos anos. Não há registro de nenhum outro trabalho seu posterior em Relatividade Geral.

\section{Buracos negros}

A elucidação completa do significado da solução (2) demoraria décadas. Nos anos seguintes à morte de Schwarzschild, até mais ou menos o final da década de 20, as principais análises concentravam-se nas implicações observacionais de (22). Destacam-se, neste período, os trabalhos do grande astrônomo britânico Arthur Eddington, famoso por ser o responsável pela expedição de 1919 que observou um eclipse solar no hemisfério sul, mais precisamente no arquipélago de São Tomé e Príncipe, na costa africana, e na cidade de Sobral ${ }^{11}$ no Ceará, que confirmou uma previsão da Relatividade Geral que pode ser obtida a partir da solução de Schwarzschild: o desvio da luz pela ação gravitacional de corpos massivos. Foi essa expedição, a primeira envolvendo conjuntamente cientistas britânicos e alemães após a Primeira Guerra, que deu fama mundial a Einstein [8].

Eddington talvez tenha sido o primeiro a questionar consistentemente a natureza da "singularidade" presente para $r=r_{S}$ na solução (2). O próprio Schwarzschild, aparentemente, não dera muita importância à singularidade, afinal a solução Newtoniana (1) também possui uma singularidade em $r=0$, mas isso não vem a ser um problema para sua in-

\footnotetext{
${ }^{11}$ Sobre a história e alguns impactos no Brasil desta expedição, ver 3941
} 
terpretação e utilização para qualquer $r>0$. Há, porém, uma diferença fundamental entre os dois casos. Enquanto no caso Newtoniano essa singularidade pode ser envolta numa superfície de área arbitrariamente pequena, isso não ocorre na solução de Schwarzschild. A singularidade $r=r_{S}$ na Relatividade Geral não corresponde, como no caso newtoniano, a um ponto no espaço, mas a uma esfera de área

$$
A=4 \pi r_{S}^{2}=16 \pi \frac{G^{2} M^{2}}{c^{4}} .
$$

Ao contrário do caso newtoniano, a solução de Schwarzschild não nos permite confinar a singularidade numa região espacial arbitrariamente pequena e, portanto, é muito mais difícil evitar sua incorporação nas análises físicas. Deve-se, obrigatoriamente, levar em conta essas singularidades nos problemas, por exemplo, do movimento dos corpos sob a ação gravitacional de (2). Algumas destas análises foram adiantadas por Droste em seu artigo de 1916 [36]. Por outro lado, o raio de Schwarzschild é tipicamente muito pequeno. Para o caso do sol, por exemplo, que possui uma massa de aproximadamente $2 \times 10^{30} \mathrm{~kg}$, seu raio de Schwarzschild é de aproximadamente 3 $\mathrm{km}$. O raio de Schwarzschild da Terra não chega a $1 \mathrm{~cm}$. Em outras palavras, o raio de Schwarzschild é tipicamente minúsculo se comparado a corpos astrofísicos usuais, estando, nesses casos, localizado sempre no interior desses corpos, onde não se espera que as soluções (1) e (2) sejam mais válidas. A elucidação da natureza da singularidade $r=r_{S}$ iria requerer um estudo mais aprofundado dos detalhes internos e de formação dos corpos esféricos homogêneos que originam os campos gravitacionais em questão. Nesse contexto, um estudante brilhante de Eddington na Universidade de Cambridge, o indiano Subrahmanyan Chandrasekhar - Chandra 12 como era conhecido - tem um papel fundamental. Cabe aqui um comentário acerca desses dois personagens. Terminariam em uma encarniçada disputa pública, precisamente sobre a interpretação da solução de Schwarzschild como buracos negros e seu papel na evolução de uma estrela [42], que, como quase sempre ocorre nessas situações, terminou em desvantagem para o estudante, mesmo ele tendo razão neste caso em particular.

\footnotetext{
${ }^{12} \mathrm{Em}$ sua homenagem, assim foi batizado o mais importante telescópio de raios X da NASA em órbita atualmente, ver http://chandra.harvard.edu/
}

Uma contribuição fundamental ao entendimento da natureza da singularidade $r=r_{S}$ foi dada por R. Openheimer e seus colaboradores nos anos imediatamente anteriores a Segunda Guerra Mundial [43]. Estes estudos deram forma ao entendimento moderno do colapso gravitacional, que fora, de fato, inicialmente proposto por Chandra e refutado com certa violência por seu orientador, Eddington. A ideia é que determinadas configurações fisicamente razoáveis de matéria auto-gravitante poderiam colapsar, implodir devido a própria atração gravitacional. É exatamente o que ocorre, por exemplo, para o caso tratado por Schwarzschild em seu segundo artigo [21, o caso de um corpo esférico homogêneo de raio externo inferior a $\frac{9}{8} r_{S}$. Nesta situação, as tensões no centro da distribuição de matéria divergem, e nenhuma força de reação física será capaz de sustentar a distribuição, que acabará colapsando sobre ação do próprio peso, literalmente implodindo, concentrando-se em regiões cada vez mais pequenas e acabando finalmente em um único ponto, $r=0$. Este colapso total da matéria, que nunca foi aceito por Eddington e se transformou no principal motivo das polêmicas e brigas públicas com Chandra, deixará inexoravelmente o raio de Schwarzschild exposto, e portanto passível de ser explorado fisicamente. Openheimer identificou corretamente a superfície $r=r_{S}$ como uma superfície de redshift (desvio para o vermelho) máximo [44 45] . Por um efeito semelhante ao efeito Doppler 13 na Relatividade Geral a freqüência de uma onda de luz pode variar de acordo com o potencial gravitacional local. A luz emitida na superfície $r=r_{S}$ da solução de Schwarzschild sofre um desvio máximo para o vermelho, quer dizer, sua freqüência, qualquer que seja, é reduzida a valores arbitrariamente baixos. Na prática, isto significa que nenhum sinal luminoso emitido da superfície $r=r_{S}$ conseguirá, por exemplo, chegar a observadores distantes, em $r \gg r_{S}$. Estas configurações foram denominadas frozen stars por Openheimer. Seriam corpos frios, de certa forma "mortos", e não seria possível detectar nenhuma emissão eletromagnética oriunda deles (por isso mesmo, em particular, serão corpos frios

\footnotetext{
${ }^{13}$ Efeito ondulatório clássico, no qual a freqüência da onda se altera devido ao movimento relativo entre a fonte e o observador, perfeitamente ilustrado pela situação em que a sirene de uma ambulância em movimento soa mais aguda quando ela se aproxima de um observador em repouso, e torna-se mais grave ao se afastar.
} 
para os observadores distantes, já que o calor se irradia efetivamente como ondas eletromagnéticas).

Situações que envolvem um raio de Schwarzschild exposto, fora da distribuição de matéria que origina o campo gravitacional, necessariamente envolvem corpos de densidades muito altas. Seria como colocar a massa do sol em um corpo de $3 \mathrm{~km}$ de raio, ou a massa da Terra em um corpo com menos de 1 $\mathrm{cm}$ de raio. Decididamente, são situações que fogem da nossa experiência cotidiana. Porém, é muito interessante notar que a possibilidade de que um corpo seja tão denso que sua atração gravitacional superficial seja tão intensa que não permitiria que sequer sinais luminosos fossem emitidos de sua superfície, já havia sido considerada no contexto newtoniano.

Há aqui também um pequeno conflito na atribuição dos créditos pelas ideias originais 46 . A possibilidade de existência no contexto newtoniano destes corpos escuros, que não emitiriam nenhuma luz devido à intensa atração gravitacional em suas superfícies, é normalmente atribuída a Pierre-Simon Laplace [47, gênio científico francês, que viria a se tornar marquês com a restauração da dinastia dos Bourbons após a queda de Napoleão. Porém, há pelo menos um antecessor que considerou - e publicou - estudos dessa mesma natureza. Trata-se de John Michell, naturalista inglês que considerou o mesmo problema no mínimo 20 anos antes que Laplace [48]. As ideias aqui são relativamente simples, e podem ser perfeitamente entendidas a partir das bases elementares da mecânica newtoniana [49].

Consideremos, por exemplo, um planeta de massa $M$ e raio $R$, e um corpo de teste de massa $m$ muito menor que $M$, inicialmente sobre a superfície do planeta. Suponhamos, por simplicidade, que este corpo seja arremessado radialmente, para fora do planeta, com uma velocidade inicial $v$. A energia total $E$ do corpo teste, dada por

$$
E=\frac{m v^{2}}{2}-\frac{G M m}{r},
$$

é conservada em todos os instantes do movimento. A questão fundamental aqui é que a energia potencial gravitacional newtoniana $U$, o segundo termo do lado direito de (5), é uma quantidade não positiva. Seu mínimo, $U_{\min }=-\frac{G M m}{R}$, corresponde ao valor de $U$ na superfície do planeta, $r=R$, e seu máximo será $U_{\max }=0$, que corresponde ao corpo de teste infinitamente afastado, $r \rightarrow \infty$. Se a velocidade $v$ com que o corpo é lançado radialmente a partir de $r=R$ é tal que a energia total (5) ainda é negativa, em outras palavras, a energia cinética inicial $T=$ $\frac{m v^{2}}{2}$ não é suficiente para "vencer" o potencial de atração $\frac{G M m}{R}$, o corpo de afastará até um $r=r_{\max } \mathrm{e}$ então retornará a superfície, a qual por certo chegará com a mesma velocidade em módulo com a que foi lançado, já que $E$ deve ser conservada. Quanto maior $v$, enquanto $E$ for negativa, maior será $r=r_{\max }$. Ocorre que para $v \geq v_{c}$, com

$$
v_{c}^{2}=\frac{2 G M}{R},
$$

esse corpo não retornará nunca mais ao planeta, pois sua velocidade $v$ é superior à velocidade de escape $v_{c}$, permitindo que ele se livre completamente da atração gravitacional do planeta. Esta é a velocidade com que um foguete deve ser lançado desde a Terra para atingir uma trajetória balística que não retornará (trajetória não elíptica). Como é característico dos fenômenos gravitacionais, esta velocidade limite não depende da massa $m$ do corpo de teste, depende apenas da massa $M$ da fonte do campo gravitacional (o planeta).

Sabe-se desde o século XVII [27], notadamente das observações astronômicas do astronômo dinamarquês Ole Rømer, que a luz se propaga a uma velocidade de aproximadamente $300.000 \mathrm{~km} / \mathrm{s}$. Denotandose por $c$ a velocidade da luz, temos de (6) que um corpo de massa $M$ e raio $R$ inferior, precisamente, ao raio de Schwarzschild (3), terá uma velocidade de escape na sua superfície superior a $c$. Em outras palavras, o corpo será tão denso que a luz não seria capaz de vencer a atração gravitacional em sua superfície. Nenhuma luz seria emitida por um corpo dessa natureza $a^{14}$ O raio de Schwarzschild, portanto, tem um precedente newtoniano. O conceito de um objeto frio e negro, no sentido que não emitirá nenhuma radiação eletromagnética, introduzido por Openheimer, tem um equivalente newtoniano. Esta ideia ainda primitiva de "buraco negro no céu" não foi bem aceita. Contou, em particular, com a oposição do próprio Einstein, que em 1939 publicou um artigo [50] no qual argumentava que o colapso gravitacional não terminaria inexoravelmente na exposição do raio de Schwarzschild e, portanto, na formação de buracos negros. Einstein estava tão

\footnotetext{
${ }^{14} \overline{\text { Obviamente, temos aqui }}$ um pequeno problema que não pode ser resolvido satisfatoriamente no contexto newtoniano: se para a luz valeria a expressão (5) como energia total conservada. Esta análise necessariamente requer conceitos relativísticos, mas estes argumentos newtonianos simples apontam na direção correta.
} 
seguro de seu argumento, o qual com o passar do tempo se mostraria equivocado, que conclui este artigo declarando:

The essential result of this investigation is a clear understanding as to why the "Schwarzschild singularities" do not exist in physical reality.

Não houve consenso à época sobre a possibilidade física real de existência desses objetos, e essa situação perdurou por quase duas décadas.

A Segunda Guerra Mundial afetou, obviamente, o desenvolvimento das ciências não diretamente ligadas aos esforços bélicos dos países envolvidos. Openheimer, por exemplo, dedicou-se ao Projeto Manhattan, que culminaria na construção das primeiras bombas atômicas, e muito provavelmente não teve mais tempo para prosseguir com seus estudos de Relatividade Geral. Vários outros físicos tiveram experiências semelhantes. Com o final da Segunda Guerra, a vida científica tende a voltar ao normal nos países centrais envolvidos nos conflitos, mas é evidente que há um certo desinteresse pela Relatividade Geral, que acaba relegada a um segundo plano quando comparada, por exemplo, as florescentes Eletrodinâmica Quântica e Física do Estado Sólido. A Relatividade Geral no pós-guerra se transforma numa teoria altamente especulativa, nem sempre bem formulada matematicamente e um tanto vazia de conteúdo físico, e por isso pouco atraente do ponto de vista científico. Essa fase um tanto obscura, de esquecimento, foi registrada sarcasticamente em uma carta que o célebre físico americano Richard Feynman escreveu a sua esposa Gweneth, enquanto assistia a uma conferência de Relatividade Geral em Varsóvia, em 1962. Dizia à sua esposa, textualmente [51]:

... I am learning nothing. Because there are no experiments this field is not an active one, so few of the best men are doing work in it (...) whenever anyone asks me a question or starts to tell me about his "work". The "work" is always:

1. completely un-understandable,

2. vague and indefinite,

3. something correct that is obvious and self evident ...

4. claim based on the stupidity of the author ...

5. an attempt to do something probably impossible, but certainly of no utility,

6. just plain wrong.

... Remind me not to come to any more gravity conferences!
O curioso é que é justamente nessa época, em que Feynman registra essas suas péssimas impressões, que a Relatividade Geral começa a renascer como teoria física.

\subsection{O renascimento: os buracos negros como objetos reais da Natureza}

O interesse científico na área renasce basicamente no final dos anos 50. Uma contribuição seminal foi dada por David Finkelstein em 1958 [52], resolvendo de maneira definitiva o impasse que reinava desde os anos 30 sobre a possível realização física do raio de Schwarzschild $r_{S}$. Seu trabalho possibilita a interpretação correta da esfera de raio $r=r_{S}$ como uma espécie de membrana unidirecional que só permitira o cruzamento de trajetórias físicas, incluindo as da luz, de fora para dentro. Quer dizer, a esfera de raio $r_{S}$ funciona como uma espécie de fronteira entre duas regiões, sendo a região externa completamente desconectada da interior, sem qualquer possibilidade de comunicação de dentro para fora. Nascia assim o conceito de "horizonte de eventos", que de fato é o que distingue e define um buraco negro.

Contudo, a atividade retorna de maneira mais vigorosa com os estudos das implicações astrofísicas dessas novas estruturas espaço-temporais recémdescobertas. Como estamos em plena Guerra Fria, é claro que havia uma enorme polarização na comunidade científica, e as principais atividades se davam em torno das escolas soviética, liderada pelo brilhante físico Yakov Borisovich Zel'dovich, e da americana, sob orientação de John Archibald Wheeler [43. Lev Landau, um dos grandes do Século XX, e seu grupo, também dedicou vários estudos à Relatividade Geral nessa época. Wheeler é um personagem a parte [53]. Teve uma longa e produtiva carreira, tendo contribuído de maneira profunda a diferentes áreas da Física ao longo do Século XX. Conhecido por suas claras e divertidas analogias e por ser um grande frasista, foi considerado por muito tempo como o responsável por ter cunhado a expressão "buraco negro" para a solução de Schwarzschild. Efetivamente, Wheeler difundiu essa denominação mas, contudo, a verdadeira responsável pela introdução do termo na literatura foi uma jornalista, Ann E. Ewing [54], que ouvira em um congresso da Sociedade Americana para o Avanço da Ciência, em 1964, a expressão black holes in space e a reproduziu em um artigo de divulgação [55] sem, porém, identificar a fonte $[56$. 
Wheeler e seus colaboradores, quase todos seus alunos em Princeton, foram fundamentais para organizar os estudos modernos sobre buracos negros, dando origem a uma escola que até hoje perdura. Além de compilarem uma vasta gama de possíveis previsões experimentais e observacionais dos buracos negros, como as que foram comprovadas este ano com as ondas gravitacionais, também sistematizaram todo o conhecimento teórico acerca desses objetos fascinantes. Desses estudos, em particular, infere-se que os buracos negros são objetos de extrema simplicidade. Analisando a solução de Schwarzschild (2), vemos que ela depende apenas de um único parâmetro, $r_{S}$, que por sua vez depende unicamente da massa total $M$ do buraco negro (além, obviamente, da constante $G$ de Newton e da velocidade da luz $c$ ). Sabemos, hoje, que a solução de Schwarzschild é o elemento mais simples de uma classe mais ampla, mas mesmo assim muito simples. O buraco negro mais geral que pode existir na teoria da Relatividade Geral é caracterizado por outros dois parâmetros além de sua massa: seu momento angular total $J$ e sua eventual carga elétrica $Q$. $\mathrm{Na}$ Natureza, a matéria apresenta-se predominantemente como neutra eletricamente, isto é, tipicamente composta a partir de cargas elementares negativas e positivas em números mais ou menos iguais. Isto significa que não se espera, em situações astrofísicas, buracos negros com carga elétrica apreciável, pois a matéria que o originou muito provavelmente era neutra. Do ponto de vista matemático, porém, existe a possibilidade de um buraco negro carregado eletricamente. Já o caso do momento angular é diferente. Os mecanismos físicos mais comuns para a criação de um buraco negro envolvem distribuições de matéria que giram e, portanto, têm momento angular não nulo, e parte deste momento angular certamente será transferido ao buraco negro que eventualmente surja após um colapso gravitacional. Em outras palavras, em um colapso gravitacional, não importa que tipo de distribuição de matéria esteve envolvida, o estágio final será um buraco negro completamente caracterizado por só três parâmetros: massa $M$, momento angular $J$ e, possivelmente, uma pequena carga elétrica $Q$. Visto de fora, para observadores externos ao horizonte de eventos $r=r_{S}$, todos os buracos negros são iguais, não possuirão nenhum outro adereço que os possa identificar além desses três parâmetros físicos. Wheeler deu o nome de "cabelos" a estes possíveis adereços e, por exemplo, em seu conhecido e bastante completo livro texto sobre Relatividade Geral [57], descreve, já na introdução, os fortes vínculos impostos pelas equações de campo na Teoria da Relatividade Geral da seguinte maneira:

The field equation (...) governs the collapse of a star to form a black hole; it determines uniquely the external spacetime geometry of a black hole ("a black hole has no hair")...

Os black hole hairs tornaram-se um fértil campo de pesquisa. A possibilidade de que objetos astrofísicos de tamanho apreciável, de várias massas solares, por exemplo, possam ser completamente caracterizados por apenas três parâmetros, como se tratassem de partículas elementares, é fascinante e tem diversas implicações teóricas e observacionais. Neste caso, porém, estamos nos aproximando muito das pesquisas atuais, sem o tempo necessário para que os fatos e ideias se assentem completamente e nos permitam uma análise minimamente isenta do ponto de vista histórico.

\subsection{Buracos negros e ondas gravitacionais}

Como já foi dito, a Teoria da Relatividade Geral de Einstein prevê a existência de ondas gravitacionais. De fato, esta deve ser uma previsão de qualquer teoria que rejeite interações instantâneas à distância, como as presentes na Gravitação Universal de Newton. Se uma interação da Natureza não é instantânea, a primeira pergunta é como ela se propaga. Na Relatividade Geral, a interação gravitacional se propaga com a mesma velocidade que a luz, algo que por certo também foi confirmado pelas recentes detecções. As estimativas iniciais, feitas pelo próprio Einstein, apontavam corretamente que as amplitudes típicas das ondas gravitacionais seriam minúsculas, talvez não detectáveis na prática. Porém, havia uma outra disputa: se estas ondas existiriam efetivamente na Natureza, mesmo que com amplitudes mínimas, ou se seriam uma mera construção matemática, sem realidade física. Eddington era um dos que duvidavam do conteúdo físico das ondas gravitacionais. Sua posição a respeito foi eternizada por sua frase jocosa acerca da propagação das ondas gravitacionais 58

Gravitational waves propagate at the speed of thought. 
Em 1918, Einstein [59] publica o primeiro artigo inteiramente dedicado às ondas gravitacionais. Vários outros se sucederam, alguns já na sua época em Princeton (ver também [6]). Seu já famoso desentendimento com o editor do Physical Review da época, John T. Tate, se deu exatamente por problemas, na ótica de Einstein, no tratamento editorial dado a alguns de seus trabalhos sobre ondas gravitacionais. $\mathrm{O}$ levantamento do sigilo editorial do Physical Review, após passados 70 anos e sem nenhum dos protagonistas ainda vivos, esclareceu este impasse [60], e a conclusão não foi das mais edificantes para a imagem de Einstein.

O ponto fundamental aqui é que o entendimento mais profundo das ondas gravitacionais, que deixou absolutamente claro que elas têm efetivamente um conteúdo físico, envolve precisamente a solução de Schwarzschild. Da mesma maneira que uma onda é criada quando perturbamos o equilíbrio da superfície da água em um tanque, ondas gravitacionais surgirão e se propagarão quando perturbamos a solução de Schwarzschild. As ondas gravitacionais são, portanto, pequenos desvios no campo gravitacional esfericamente simétrico correspondente à solução (2), que de acordo com a Relatividade Geral devem se propagar com a velocidade da luz. A solução de Schwarzschild (2) tem propriedades matemáticas (especificamente, o fato de ser assintoticamente plana) que permitem a identificação e interpretação de propriedades físicas como momento e energia transportados pelas ondas gravitacionais, resolvendo de maneira inequívoca as questões acerca da realidade física destas ondas. São tão físicas como qualquer outro fenômeno ondulatório encontrado na Natureza, sendo caracterizadas pelos mesmas quantidades como frequência, comprimento de onda e velocidade de propagação. Sendo assim, são passíveis de serem detectadas experimentalmente, uma vez vencido o desafio de lidar com quantidades de amplitudes mínimas, como fez o consórcio LIGO-Virgo $1,2[15$. Além de permitir a interpretação física definitiva das ondas gravitacionais, a solução de Schwarzschild também tem papel importante na descrição dos eventos que geram os sinais gravitacionais que podem ser detectados com maior freqüência na Terra: os fenômenos envolvendo fusão de buracos negros.

A solução de Schwarzschild, assim como suas generalizações contendo momento angular (solução

\footnotetext{
${ }^{15}$ Para uma exposição mais técnica, ver 61
}

de Kerr) ou carga elétrica (Reissner-Nordström ou Kerr-Newman), corresponde a uma solução da Relatividade Geral para o problema de um corpo. Não existe solução exata explícita para o problema de dois corpos em Relatividade Geral. Assim, se quisermos descrever, na Relatividade Geral, uma configuração que corresponda, por exemplo, a um sistema binário, onde dois corpos de massas comparáveis se movem sob ação gravitacional mútua, necessariamente temos que utilizar aproximações ou algum tipo de solução computacional para as equações de Einstein. A fusão de buracos negros que originou os sinais detectados este ano correspondem a um sistema binário de buracos negros que gradativamente perdem energia, emitida como ondas gravitacionais, e com isso vão se aproximando, seguindo órbitas que espiralam e, finalmente, coalescem e dão origem a um único buraco negro. No caso do primeiro evento detectado este ano [3], por exemplo, o sistema binário original era composto por dois buracos negros de massas iguais a 29 e 36 massas solares. O buraco negro final tem 62 massas solares. As 3 massas solares faltantes foram irradiadas, quase que totalmente, em ondas gravitacionais! É como se 3 sois de repente desaparecessem, e suas energias de repouso (a gigantesca quantidade dada pela fórmula $E=m c^{2}$ ) se dissipassem no universo como ondas gravitacionais. A descrição realista desse sistema envolve um considerável esforço computacional, algo que seria impossível algumas décadas atrás. O estado-da-arte na área envolve sofisticados recursos de hardware e software, mas as origens dos conceitos centrais inequivocamente remontam à solução de Schwarzschild (2), que encantou Einstein por sua simplicidade 100 anos atrás.

\section{Comentários Finais}

Após a detecção direta das ondas gravitacionais, é natural que se pergunte, neste contexto que envolve buracos negros, se há alguma possibilidade de obervação direta destes objetos. Isto corresponderia, de fato, a observação de um horizonte de eventos, a estrutura espaço-temporal que funciona como uma membrana unidirecional. Claramente, não se espera sinais detectáveis do interior do buraco negre ${ }^{16}$ Pode-se, porém, detectar o sinal da matéria

\footnotetext{
${ }^{16}$ Isto, efetivamente, não é completamente certo. Curiosamente, descobriu-se nos anos 70 que os buracos negros, afinal, não são negros de fato, mas emitem um pequeno brilho, a cha-
} 
que cruza o horizonte. E há, justamente em nossa galáxia, uma possibilidade dessas. Trata-se de um buraco negro gigantesco, com cerca de 4 milhões de massas solares, no centro de nossa galaxia, na região do céu conhecida como Sagitário $A^{*}$. A possibilidade de existência de buracos negros gigantes no centro ativo de galáxias vem sendo aventada desde o final dos anos 60 [62]. Há uma grande quantidade de galáxias com núcleos ativos, porém, devido à proximidade, Sagitário $A^{*}$ é considerada como a mais promissora possibilidade de observação direta de um buraco negro, e diversos telescópios atualmente varrem essa região do céu, e várias estrelas com trajetórias próximas ao provável buraco negro já foram identificadas e estão sendo constantemente seguidas. O cruzamento do horizonte por uma dessas estrelas ou qualquer material que eventualmente emane delas deixará um sinal que poderá ser inequivocamente identificado, e há intensos trabalhos teóricos e observacionais nessa campo atualmente. A saga que se iniciou há um século com Schwarzschild, está ainda longe do seu final.

Apesar de sua vida ter sido bastante breve, Schwarzschild pode receber diversos prêmios e distinções e desfrutar do reconhecimento de seus pares [14]. Postumamente, foi homenageado também em diversas situações. Destacam-se o nome dado nos anos 60 para o maior observatório astronômico da Alemanha, Karl-Schwarzschild-Observatorium, atualmente Thüringer Landessternwarte Karl Schwarzschild Tautenburg, localizado em Tautenburg, próximo a Jena, e a instituição da Medalha Karl Schwarzschild, premiação anual da Sociedade Astronômica Alemã para eminentes astrônomos e astrofísicos. Seu filho Martin Schwarzschild foi o primeiro a recebê-la, em 1959 .

\section{Agradecimentos}

O autor reconhece e agradece o contínuo suporte financeiro recebido do Conselho Nacional de Desenvolvimento Científico e Tecnológico - CNPq (processos 304378/2014-3 e 441349/2014-5) e da Fundação de Amparo à Pesquisa do Estado de São Paulo - FAPESP (processo 2013/09357-9). Agradece também, muito especialmente, os organizadores e participantes da série de colóquios "Convite à Física", do

mada radiação de Hawking, chamada assim devido ao célebre físico inglês que a propôs. Contudo, para buracos negros de dimensões astrofísicas, este brilho seria completamente imperceptível.
Departamento de Física Matemática do Instituto de Física da USP. Este texto foi a base do colóquio lá apresentado, em abril de 2016, como celebração aos 100 anos dos buracos negros.

\section{Referências}

[1] http://www.ligo.caltech.edu/

[2] http://www.ego-gw.it/

[3] B.P. Abbott, LIGO Scientific Collaboration and Virgo Collaboration, Phys. Rev. Lett. 116, 061102 (2016).

[4] E. Berti, Physics 9, 17 (2016)

[5] G.E.A. Matsas, Ciência Hoje 334, 11 (2016).

[6] C.V. Leite, Ciência Hoje 334, 12 (2016).

[7] B.P. Abbott, LIGO Scientific Collaboration and Virgo Collaboration, Phys. Rev. Lett. 116, 241103 (2016).

[8] W. Isaacson, Einstein - Sua Vida, Seu Universo (Companhia das Letras, São Paulo, 2007).

[9] P.G. Ferreira, The perfect Theory (Hughton Mifflin, Boston, 2014).

[10] Press Release: The 1993 Nobel Prize in Physics, disponível em http://www.nobelprize.org/

[11] J.M. Weisberg, J.H. Taylor and L.A. Fowler, Scientific American 245, 74 (1981).

[12] K. Schwarzschild, Sitzungsber. Preuss. Akad. Wiss. Berlin (Math. Phys.) 1916, 189 (1916). Tradução para o inglês disponível em http://arxiv.org/ abs/physics/9905030

[13] E. Hertzsprung, Astrophysical J. 45, 285 (1917).

[14] H.H. Voigt (ed), Karl Schwarzschild: Gesammelte Werke/Collected Works: Volume 1 (German and English Edition) (Springer, Berlin, 1992).

[15] K. Schwarzschild, Astron. Nachr. 124, 211 (1890), reproduzido em [14].

[16] K. Schwarzschild, Astron. Nachr. 124, 215 (1890), reproduzido em 14.

[17] K. Schwarzschild, Neue Annalen der K. Sternwarte in Bogenhausen bei Muenchen 3, 231 (1898).

[18] K. Schwarzschild, Sitzungsber. Preuss. Akad. Wiss. Berlin 1920, 37 (1920).

[19] F. Stern, O Mundo Alemão de Einstein (Companhia das Letras, São Paulo, 2004).

[20] To the Civilized World, by Professors of Germany, The North American Review 210, 284 (1919). Original alemão publicado em 4 de outubro de 1914, com título Der Aufruf "An die Kulturwelt!" (Manifesto "ao mundo civilizado!" - tradução livre do autor), assinado por 93 proeminentes cientistas.

[21] K. Schwarzschild, Preuss. Akad. Wiss. Berlin (Math. Phys.) 1916, 424 (1916).

[22] A. Einstein, Sitzungsber. Preuss. Akad. Wiss. Berlin (Math. Phys.), 1915, 315 (1915).

[23] A. Einstein, Preuss. Akad. Wiss. Berlin (Math. Phys.) 1915, 778 (1915); 1915, 799 (1915). 
[24] A. Einstein, Preuss. Akad. Wiss. Berlin (Math. Phys.) 1915, 831 (1915).

[25] A. Einstein, Preuss. Akad. Wiss. Berlin (Math. Phys.) 1915, 844 (1915).

[26] A. Einstein, Annalen der Physik 1916, 49, 769 (1916)

[27] E. Abdalla e A. Saa, Cosmologia: Dos Mitos ao Centenário da Relatividade (Edgard Blucher, São Paulo, 2010)

[28] I. Newton, Philosophiae Naturalis Principia Mathematica (Londres, Jussu Societatis Regiae, 1686). Versão inglesa disponível no Projeto http://en. wikisource.org

[29] F.T. Falciano, Ciência Hoje 334, 60 (2016).

[30] A. Saa, Ciência Hoje 255, 74 (2008).

[31] A. Saa, Ciência Hoje, a ser publicado.

[32] K. Schwarzschild, Vierteljahrsschrift der Astronomische Gesellschaft 35, 337 (1900). Tradução para o inglês em On the Permissible Numerical Value of the Curvature of Space, Abraham Zelmanov J. 1, 64 (2008).

[33] J. Gray, in: Non-Euclidean Geometries: János Bolyai Memorial Volume, editado por A. Prékopa e E. Molnár, Mathematics and Its Applications 581, 61 (2006).

[34] J. Gray, in: The Stanford Encyclopedia of Philosophy editado por Edward N. Zalta, disponível em http: //plato.stanford.edu

[35] A. Zee, Einstein Gravity in a Nutshell (Princeton University Press, Princeton, 2013).

[36] J. Droste, Kon. Ak. Wetensch. Amsterdam 25, 163 (1916). Versão em inglês publicada em Proc. Acad. Sc. Amsterdam 19, 197 (1917).

[37] T. Rothman, General Relativity and Gravitation 34, 1541 (2002).

[38] J. Droste, Het zwaartekrachtsveld van een of meer lichamen volgens de theorie van Einstein. Dissertation, Universidade de Leiden, 1916.

[39] M.C. de Lima and L.C.B. Crispino, Int. J. Mod. Phys D 25, 1641002 (2016).

[40] L.C.B. Crispino and M.C. de Lima, Phys. Perspect doi: http://dx.doi.org/10.1007/s00016016-0190-3 (2016).

[41] L.C.B. Crispino e M.C. de Lima, Rev. Bras. Ensino Fís., a ser publicado.

[42] A.I. Miller, Empire of the Stars: Obsession, Friendship, and Betrayal in the Quest for Black Holes (Houghton Mifflin Harcourt, Boston, 2005).

[43] K.S. Thorne, Black Holes and Time Warps: Einstein's Outrageous Legacy (W.W. Norton \& Company, New York, 1995).

[44] D. Vanzella e G. A. Matsas, Buracos Negros: Rompendo os Limites da Ficção (Vieira \& Lent, Rio de Janeiro, 2008).

[45] J.E. Horvath e P.S. Custodio, Os Buracos Negros na Ciência Atual: Um Brevíssimo Manual Introdutório (Livraria da Física, São Paulo, 2012).
[46] C. Montgomery, W. Orchiston and I. Whittingham, J. Astronomical History and Heritage 12, 90 (2009).

[47] P.S. Laplace, Proof of the theorem, that the attractive force of a heavenly body could be so large, that light could not flow out of it (Cambridge University Press, Cambridge, 1973), tradução ao inglês do original francês apresentada no Apêndice A de S.W. Hawkinge e G.F.R. Ellis, The large scale structure of space-time.

[48] J. Michell, Phil. Transc. Roy. Soc. of London 74, 35 (1783).

[49] R.R. Machado e A.C. Tort, Rev. Bras. Ensino Fís. 38, e2314 (2016).

[50] A. Einstein, Ann. Math. 40, 922 (1939).

[51] Richard P. Feynman, What Do You Care What Other People Think?: Further Adventures of a Curious Character, edited by R. Leighton (W.W. Norton \& Company, New York, 2001).

[52] D. Finkelstein, Phys. Rev. 110, 965 (1958).

[53] K.W. Ford and J.A. Wheeler, Geons, Black Holes, and Quantum Foam: A Life in Physics (W.W. Norton \& Company, New York, 2000).

[54] Obituário publicado em 1 de Agosto de 2010, no jornal The Washington Post http://www washingtonpost.com/.

[55] A.E. Ewing, Science News Letter 85, 39 (1964).

[56] T. Siegfried, 50 Years Later, It's Hard to Say Who Named Black Holes, disponível em https://www. sciencenews.org/

[57] C.W. Misner, K.S. Thorne and J.A. Wheeler, Gravitation (W.H. Freeman, New York, 1973).

[58] D. Kennefick, Traveling at the Speed of Thought: Einstein and the Quest for Gravitational Waves (Princeton University Press, Princeton, 2007).

[59] A. Einstein, Preuss. Akad. Wiss. Berlin (Math. Phys.) 1918, 154 (1918).

[60] D. Kennefick, Physics Today 58, 43 (2005).

[61] M. Cattani e J.M.F. Bassalo, Rev. Bras. Ensino Fís., a ser publicado.

[62] F. Melia, The Galactic Supermassive Black Hole (Princeton University Press, Princeton, 2007). 